\title{
The gut microbiota of critically ill patients: first steps in an unexplored world
}

\author{
Étienne Ruppé ${ }^{1,2}$,Thiago Lisboa ${ }^{3,4}$ and François Barbier ${ }^{5^{*}}$
}

The gut microbiota is a complex ecosystem encompassing all bacteria, fungi, archaea, viruses, and protozoa that colonize the intestinal tract, reaching in healthy humans an estimated total of $3.10^{13}$ microorganisms that roughly equals the number of host cells [1]. Bacterial commensals are divided up into seven main phyla that are physiologically dominated by Firmicutes and Bacteroidetes (Fig. 1), although their richness and diversity may exhibit substantial inter- as intra-individual variations depending on genetic, dietary, and environmental factors [2]. Several host-benefic functions have been linked to a "normal" gut microbiota and its symbiotic relationship with the intestinal mucosa, including contributions to hormonal homeostasis, carbohydrate and biliary acid metabolism, vitamin synthesis, anti-inflammatory pathways, and immune regulation [3]. Of note, most enteric bacteria are unculturable or exclusively grow under strict anaerobic conditions that are very demanding to achieve in experimental laboratories, which justifies the need for non-culture-based assays and bioinformatics to investigate the composition of this microbial community. Two main methods based on nucleic acid sequencing are currently available. The first one is $16 \mathrm{~S}$ profiling, which relies on PCR-based amplification and sequencing of a fraction of the bacterial ubiquitous $16 \mathrm{~S}$ rRNA-encoding gene. This approach is simple and cheap-less than 100 USD per sample; however, bacterial identifications are often limited to high taxonomic levels. The second one, referred to as shotgun metagenomics, consists in sequencing the whole DNA of a given sample without prior amplification. This method allows more accurate taxonomic assignments

\footnotetext{
*Correspondence: francois.barbier@chr-orleans.fr

${ }^{5}$ Service de Médecine Intensive et Réanimation, Hôpital de la Source, Centre Hospitalier Régional d'Orléans, Orléans, France

Full author information is available at the end of the article
}

(down to species level, including for non-bacterial components of the microbiota) while providing information on resistance or virulence genes content. Yet, associated costs-more than 300 USD per sample-and the complex data analyses that it requires hamper the use of shotgun metagenomics in large clinical studies.

Intestinal dysbiosis could be defined as an altered dialogue between enteric bacteria and the host's cells due to disrupted microbiota diversity, usually associated with the dominance of a given taxon. Over the past decade, convincing evidence has emerged to support a promoting role for intestinal dysbiosis in the pathogenesis of diverse conditions such as metabolic diseases, autoimmunity, inflammatory bowel diseases, neurocognitive impairment, or neoplasms [3]. More recently, several studies have shed light on the functions and architectural shifts of this ecosystem in the specific context of critical illness. Indeed, a variety of both exogenous and patientrelated factors may lead to ICU-acquired dysbiosis, with antimicrobial exposure, use of proton pump inhibitors or depressors of gastrointestinal transit (e.g., opioids), artificial nutrition, sepsis, shock, or bowel ischemia being examples among others [4]. These, along with differences in baseline features, imply that two patients cannot harbor the same microbiota at a given time of their ICU stay. Overall, loss of diversity is commonly observed and may combine a deep depletion or even the complete disappearance of potential "health-promoting" commensal genus (e.g., Faecalibacterium, Ruminococcus, or Pseudobutyrivibrio) and the overgrowth of a pathogenic and normally sub-dominant taxon (e.g., Enterococcus or Enterobacteriaceae) [5-7]. Still, whether intestinal dysbiosis is an independent predictor of poor outcome rather than a mere surrogate marker of severity or prolonged stay remains somewhat speculative owing to inter-study discrepancies in terms of case-mix, prior antimicrobial

\section{Springer}




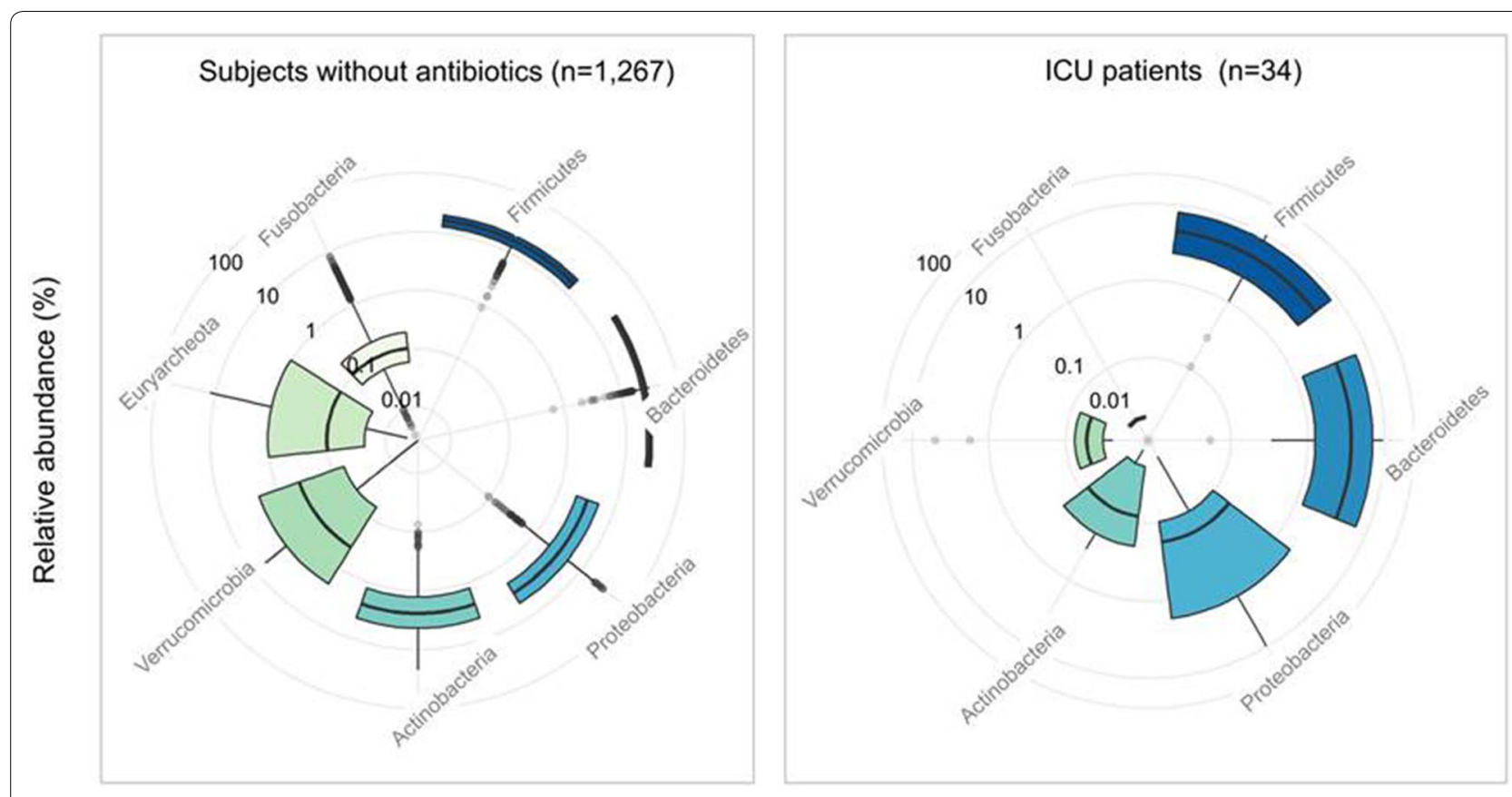

Firmicutes: Clostridium, Lactobacillus, Enterococcus, Streptococcus

Bacteroidetes: Bacteroides, Prevotella

Proteobacteria: Enterobacteriaceae, Pseudomonas, Acinetobacter

Actinobacteria: Corynebacterium, Actinomyces, Bifidobacterium

Verrucomicrobia: Akkermansia

Euryarcheota: not bacteria but Archeae .

Fusobacteria: Fusobacterium

Fig. 1 Box plot representation of the seven most abundant bacterial and archeal phyla (with examples of genera) of the human intestinal microbiota: subjects without recent antibiotic exposure and ICU patients. Data from subjects without recent antibiotic exposure were extracted from the genus profile table accessible at http://meta.genom ics.cn/meta/dataTools (1267 samples from Danish, Spanish, American, and Chinese subjects) [2]. Data from ICU patients were obtained from Lankelma et al. [5]. Relative abundance refers to the ratio between the number of reads assigned to the phylum and the total number of reads obtained after sequencing. The $y$-axis scale is $\log 10-$ scaled for clarity

exposure in enrolled patients, timing of stool sampling during the ICU stay, and analytical methods. That most of studies were conducted in ICUs implementing routine selective digestive decontamination further complicates the interpretation of available data. Nonetheless, dysbiosis could impair gut barrier functions and worsen post-aggressive immunosuppression, thereby easing the occurrence of ICU-acquired sepsis and protracted multiorgan failure [4]. Also, experimental models suggest that the composition of the gut ecosystem might modulate the risk of complications such as acute respiratory distress syndrome [8], ischemia/reperfusion-related acute kidney injury [9], or sepsis-induced muscle wasting [10].

Another key point is that resident anaerobes have the capacity to prevent intestinal colonization with exogenous microorganisms via indirect mechanisms such as competition for nutrient intake or induction of a targeted immune response [11]. Interestingly, certain commensals may exert species-specific colonization resistance: Clostridium bolteae and Blautia producta act synergistically to prevent the acquisition of vancomycin-resistant enterococci (VRE) [12]; colonization with Clostridium scindens appears to protect from Clostridium difficile infection [13]; while members of the Desulfovibrio, Oscillospira, Parabacteroides, or Coprococcus genera have been associated with the absence of carriage of extendedspectrum beta-lactamase-producing Escherichia coli [14]. Of note, both $16 \mathrm{~S}$ profiling and shotgun metagenomics only address the dominant fraction of the microbiota and, therefore, do not detect pathogens unless their relative fecal abundance increases sharply, most often following antimicrobial exposure. Whether the intestinal 
dominance of a given pathogen predisposes to subsequent infections due to the same bacteria is likely but had not been previously investigated in ICU patients [15].

The paper by Freedberg et al. [16] in this issue of Intensive Care Medicine provides novel insights to appraise how the characteristics of the gut microbiota upon ICU admission may predict death or subsequent infection in critically ill patients. A total of 301 patients were prospectively included in this single-ICU study. Rectal swabs were collected at admission, selectively cultured for VRE, and exploited for microbiota analyses through $16 \mathrm{~S}$ profiling. Patients were followed for 30 days for death or cultureproven bacterial infection, these events occurring in 25\% and $41 \%$ of patients, respectively. Pneumonia and bloodstream infections accounted for most of the ICU-acquired infections. After adjustment on illness severity, VRE colonization and Enterococcus dominance (30\% and 15\% of patients, respectively) were both associated with death or all-cause infection [adjusted hazard ratio (aHR) 1.46, 95\% confidence interval (CI) CI 1.06-2.00, and aHR 1.47, 95\% CI 1.00-2.19, respectively]. Among those without VRE colonization, Enterococcus domination was also associated with excess risk of death or infection (aHR 2.13, 95\% CI 1.05-4.29). Similar results were observed when addressing death and all-cause infections separately. Imported carriage of pathogens such as E. coli, Pseudomonas spp., Klebsiella spp., and $C$. difficile was predictive of subsequent infection due to the same bacteria (as already demonstrated in culture-based studies), while VRE colonization-but not Enterococcus dominance-was associated with subsequent Enterococcus infection. It is noteworthy that overall diversity and richness of the gut microbiota at admission were not predictive of negative outcomes.

An important limitation of Freedberg's work is that analyses were restrained to rectal samples obtained at admission. Further studies should assess temporal changes in microbiome composition during the ICU stay and their impact on mortality or the risk of healthcare-associated infections. The lack of data regarding antibiotic exposure prior to ICU admission and the adjustment limited to baseline characteristics represent other significant limitations for interpretation of findings. This study, however, further supports the potential link between intestinal traits and outcomes. Although high-throughput sequencing tools will not be available at the bedside for the fine-tuning of empirical therapy in the near future, whether pathogen dominance may predict subsequent healthcare-associated infection warrants further investigations in critical care environments with different ecological issues (e.g., multidrug-resistant Gram-negative bacteria). Overall, these new-generation approaches open a wide field for future research focused on interventions that could harm (e.g., antimicrobials with biliary excretion and/or anti-anaerobe activity, or selective digestive decontamination), protect (e.g., antimicrobial stewardship initiatives or orally administered antimicrobial-adsorbing charcoals), or restore (e.g., probiotics or fecal microbiota transplantation) the gut ecosystem during critical illnesses [17-19].

\section{Author details \\ ${ }^{1}$ INSERM, IAME-UMR 1137, Université Paris Diderot-Sorbonne Paris Cité, Paris, France. ${ }^{2}$ Laboratoire de Bactériologie, Hôpital Bichat-Claude Bernard, Assistance Publique-Hôpitaux de Paris, Paris, France. ${ }^{3}$ Critical Care Department and Infection Control Committee, Hospital de Clinicas de Porto Alegre-PPG Ciencias Pneumologias, UFRGS, Porto Alegre, Brazil. ${ }^{4}$ Rede Institucional de Pesquisa e Inovação em Medicina Intensiva, Complexo Hospitalar Santa Casa, Porto Alegre, Brazil. ${ }^{5}$ Service de Médecine Intensive et Réanimation, Hôpital de la Source, Centre Hospitalier Régional d'Orléans, Orléans, France.}

\section{Funding}

None.

Compliance with ethical standards

Conflicts of interest

ER is a consultant for DaVolterra and MaaT Pharma. FB and TL declare that they have no potential conflict of interest related to the present article.

Received: 29 June 2018 Accepted: 6 July 2018

Published online: 14 July 2018

\section{References}

1. Sender R, Fuchs S, Milo R (2016) Revised estimates for the number of human and bacteria cells in the body. PLoS Biol 14:e1002533

2. Li J, Jia H, Cai X, Zhong H, Feng Q, Sunagawa S, Arumugam M, Kultima JR, Prifti E, Nielsen T, Juncker AS, Manichanh C, Chen B, Zhang W (2014) An integrated catalog of reference genes in the human gut microbiome. Nat Biotechnol 32:834-841

3. Feng Q, Chen WD, Wang YD (2018) Gut microbiota: an integral moderator in health and disease. Front Microbiol 9:151

4. Dickson RP (2016) The microbiome and critical illness. Lancet Respir Med 4(1):59-72

5. Lankelma JM, van Vught LA, Belzer C, Schultz MJ, van der Poll T, de Vos WM, Wiersinga WJ (2017) Critically ill patients demonstrate large interpersonal variation in intestinal microbiota dysregulation: a pilot study. Intensive Care Med 43(1):59-68

6. Yeh A, Rogers MB, Firek B, Neal MD, Zuckerbraun BS, Morowitz MJ (2016) Dysbiosis across multiple body sites in critically ill adult surgical patients. Shock 46(6):649-654

7. Ojima M, Motooka D, Shimizu K, Gotoh K, Shintani A, Yoshiya K, Nakamura S, Ogura H, lida T, Shimazu T (2016) Metagenomic analysis reveals dynamic changes of whole gut microbiota in the acute phase of intensive care unit patients. Dig Dis Sci 61(6):1628-1634

8. Dickson RP, Singer BH, Newstead MW, Falkowski NR, Erb-Downward JR, Standiford TJ, Huffnagle GB (2016) Enrichment of the lung microbiome with gut bacteria in sepsis and the acute respiratory distress syndrome. Nat Microbiol 1(10):16113

9. Andrade-Oliveira V, Amano MT, Correa-Costa M, Castoldi A, Felizardo RJ, de Almeida DC, Bassi EJ, Moraes-Vieira PM, Hiyane MI, Rodas AC, Peron JP, Aguiar CF, Reis MA, Ribeiro WR, Valduga CJ, Curi R, Vinolo MA, Ferreira CM, Camara NO (2015) Gut bacteria products prevent AKI induced by ischemia-reperfusion. J Am Soc Nephrol 26(8):1877-1888

10. Schieber AM, Lee YM, Chang MW, Leblanc M, Collins B, Downes M, Evans RM, Ayres JS (2015) Disease tolerance mediated by microbiome E. coli involves inflammasome and IGF-1 signaling. Science 350(6260):558-563 
11. Pamer EG (2016) Resurrecting the intestinal microbiota to combat antibiotic-resistant pathogens. Science 352(6285):535-538

12. Caballero S, Kim S, Carter RA, Leiner JM, Susac B, Miller L, Kim GJ, Ling L, Pamer EG (2017) Cooperating commensals restore colonization resistance to vancomycin-resistant Enterococcus faecium. Cell Host Microbe 21:592-602

13. Buffie CG, Bucci V, Stein RR, McKenney PT, Ling L, Gobourne A, No D, Liu $H$, Kinnebrew M, Viale A, Littmann E, van den Brink MR, Jenq RR, Taur Y, Sander C, Cross JR, Toussaint NC, Xavier JB, Pamer EG (2015) Precision microbiome reconstitution restores bile acid mediated resistance to Clostridium difficile. Nature 517(7533):205-208

14. Gosalbes MJ, Vazquez-Castellanos JF, Angebault C, Woerther PL, Ruppé E, Ferrus ML, Latorre A, Andremont A, Moya A (2015) Carriage of enterobacteria producing extended-spectrum $\beta$-lactamases and composition of the gut microbiota in an Amerindian community. Antimicrob Agents Chemother 60:507-514

15. Ruppé E, Andremont A (2013) Causes, consequences, and perspectives in the variations of intestinal density of colonization of multidrug-resistant enterobacteria. Front Microbiol 4:129
16. Freedberg DE, Zhou MJ, Cohen ME, Annavajhala MK, Khan S, Moscoso DI, Brooks C, Whittier S, Chong DH, Uhlemann AC, Abrams JA (2018) Pathogen colonization of the gastrointestinal microbiome at intensive care unit admission and risk for subsequent death or infection. Intensive Care Med. https://doi.org/10.1007/s00134-018-5268-8

17. de Gunzburg J, Ghozlane A, Ducher A, Le Chatelier E, Duval X, Ruppé E, Armand-Lefevre L, Sablier-Gallis F, Burdet C, Alavoine L, Chachaty E, Augustin V, Varastet M, Levenez F, Kennedy S, Pons N, Mentré F, Andremont A (2018) Protection of the human gut microbiome from antibiotics. J Infect Dis 217:628-636

18. Haak BW, Levi M, Wiersinga WJ (2017) Microbiota-targeted therapies in the intensive care unit. Curr Opin Crit Care 23(2):167-174

19. Ruppé E, Martin-Loeches I, Rouzé A, Levast B, Ferry T, Timsit J-F (2018) What's new in restoring the gut microbiota in ICU patient? Potential role of fecal microbiota transplantation. Clin Microbiol Infect. https://doi. org/10.1016/j.cmi.2018.05.020 